\title{
Full-length transcriptome and targeted metabolome analyses provide insights into defense mechanisms of Malus sieversii against Agrilus mali
}

\author{
Chuang Mei ${ }^{1,2}$, Jie Yang ${ }^{1}$, Peng Yan ${ }^{2}$, Ning $\mathrm{Li}^{2}{ }^{2},{\text { Kai } \mathrm{Ma}^{2} \text {, Aisajan Mamat }}^{2}$, Liqun Han ${ }^{2}$, Qinglong Dong ${ }^{1}, \mathrm{Ke} \mathrm{Mao}^{1}$ \\ , Fengwang Ma ${ }^{\text {Corresp., } 1}$, Jixun Wang ${ }^{\text {Corresp. } 2}$ \\ ${ }^{1}$ State Key Laboratory of Crop Stress Biology for Arid Areas/Shaanxi Key Laboratory of Apple, College of Horticulture, Northwest A \& F University, \\ Yangling, Shaanxi, China \\ 2 Institute of Horticultural Crops, Xinjiang Academy of Agricultural Sciences/Scientific Observing and Experimental Station of Pomology (Xinjiang), Ministry \\ of Agriculture, Urumqi, China
}

Corresponding Authors: Fengwang Ma, Jixun Wang

Email address: fwm64@nwsuaf.edu.cn, ee_wjx@163.com

Malus sieversii is the wild progenitor for many cultivars of domesticated apple and an important germplasm resource for breeding. However, this valuable species faces a significant threat in the areas north of the Tianshan Mountains in China, by the invasion of Agrilus mali, a destructive pest of apple trees belonging to the family Buprestidae. Our preliminary study has has shown that there may be resistance to this insect in $M$ sieversii plants in the field, but the corresponding molecular mechanisms remain unclear. In this study, we compared the response of insect-resistant and insect-susceptible plants of $M$ sieversii to insect feeding using full-length transcriptome and targeted metabolome. 112,103 non-chimeric full-length reads (FLNC) totaling $10.52 \mathrm{~Gb}$ of data were generating with Pacific Biosciences SingleMolecule, Real-Time (PacBio SMRT) sequencing. A total of $130.06 \mathrm{~Gb}$ data of long reads were acquired with an Illumina HiSeq. Function annotation indicated that the different expressed genes (DEGs) were mainly involved in signal transduction pathway of plant hormones and in the synthesis of compounds such as terpenes, quinones, flavonoids, and jasmonic acid. Through targeted metabolome analysis resistant strains showed higher levels of trans-cinnamic acid, caffeine and Ferulic acid after pest infestation. This study helps to decipher the transcriptional changes and related signaling paths in $M$ sieversii after an insect feeding, which lays a foundation for further research on molecular mechanisms of insect resistance in apples. 


\section{Full-length transcriptome and targeted metabolome analyses provide}

\section{2 insights into defense mechanisms of Malus sieversii against Agrilus mali}

3
Chuang Mei ${ }^{1,2}$, JieYang ${ }^{1}$, Peng $\mathrm{Yan}^{2}$, Ning $\mathrm{Li}^{2}$, Kai Ma², Aisajan Mamat ${ }^{2}$, Liqun Han², Qinlong Dong ${ }^{1}, \mathrm{Ke} \mathrm{Mao}^{1}$, Fengwang $\mathrm{Ma}^{1 *}$ and Jixun Wang${ }^{2 *}$

${ }^{1}$ State Key Laboratory of Crop Stress Biology for Arid Areas/Shaanxi Key Laboratory of Apple, College of Horticulture, Northwest A\&F University, Yangling 712100, China

${ }^{2}$ Institute of Horticultural Crops, Xinjiang Academy of Agricultural Sciences/Scientific Observing and Experimental Station of Pomology (Xinjiang), Ministry of Agriculture, Urumqi 830091, China

${ }^{*}$ Corresponding author.

Jixun Wang

E-mail address: ee_wjx@163.com

Fengwang Ma

E-mail address:fwm64@sina.com 


\section{Abstract}

20 Malus sieversii is the wild progenitor for many cultivars of domesticated apple and an important germplasm resource for breeding. However, this valuable species faces a significant threat in the areas north of the Tianshan Mountains in China, by the invasion of Agrilus mali, a destructive pest of apple trees belonging to the family Buprestidae. Our preliminary study has shown that there may be resistance to this insect in $M$ sieversii plants in the field, but the corresponding molecular mechanisms remain unclear. In this study, we compared the response of insect-resistant and insect-susceptible plants of $M$ sieversii to insect feeding using full-length transcriptome and targeted metabolome. 112,103 non-chimeric full-length reads (FLNC) totaling $10.52 \mathrm{~Gb}$ of data were generating with Pacific Biosciences Single Molecule, Real-Time (PacBio SMRT) sequencing. A total of $130.06 \mathrm{~Gb}$ data of long reads were acquired with an Illumina HiSeq. Function annotation indicated that the different expressed genes (DEGs) were mainly involved in signal transduction pathway of plant hormones and in the synthesis of compounds such as terpenes, quinones, flavonoids, and jasmonic acid. Through targeted metabolome analysis resistant strains showed higher levels of trans-cinnamic acid, caffeine and ferulic acid after pest infestation. This study helps to decipher the transcriptional changes and related signaling paths in $M$ sieversii after an insect feeding, which lays a foundation for further research on molecular mechanisms of insect resistance in apples.

Keywords: Malus sieversii; Agrilus mali; Gene discovery; Full-length transcriptome; Insect resistant 


\section{Introduction}

Malus sieversii, distributed around the Tianshan Mountains, is the wild progenitor of its kind (Duan et al. 2017). Its flowers, fruits, nutrients, and growth habits are rich in diversity (Forsline et al. 2010). These species offers unique genetic resources that can be used in apple breeding programs (Ma et al. 2017). The apple buprestid beetle (Agrilus mali) is a coleopteran wood-borer that causes fatal damage to apple trees. Its larvae, which live underneath the bark of trunks and branches, tunnel through the phloem and xylem in irregular pattern resulting in separation of the phloem from the xylem and death of the affected parts of the tree. In severe cases, the entire plant dies (Yi et al. 2016). The insect was listed as highly injurious by the Chinese government because it had killed over 600 ha of apple trees (Mei et al. 2016). Therefore, it will be difficult and/or impossible to recover many unique genes and alleles coded by $M$ sierversii if it disappeared.

When plants are attacked by insects and other herbivores, changes in signaling pathways trigger changes in proteins and metabolites in an attempt to circumvent these stresses (Barah et al. 2015; Xie et al. 2018). Morphological, biochemical, and molecular defenses can be triggered through immunological processes. Endogenous signals generated by the damaged cells also play a key role in a plant's ability to perceive herbivores, for example, through calcium channels, phosphorylation, and metabolic pathways of jasmonic acid, shikimic acid, amino acids, and terpenes (Howe et al. 2008; Liu et al. 2016; War et al. 2012). SA and ethylene can also be induced by some insect species. When insects invade, JA increase gradually.

Attacks by insects induce a systemic response in plants, which is manifested by changes in the transcriptional expression of specific functional genes and the accumulation of certain secondary metabolites (Zhuang et al. 2018). However, there are few reports on the defense to signaling system in apples affected by buprestid beetles. TIFY proteins, which are critical regulators in the JA signaling pathway, are involved in defense and stress responses during plant development, and they play a major role in plant reactions to biotic and abiotic stress (Ebel et al. 2018; Thireault et al. 2015). The TIFY protein gene family and its subfamily members improve disease and insect resistance by regulating signaling pathways of plant hormones (Thireault et al. 2015; Xia et al. 2017).

With the development of high-throughput sequencing technology, especially SMRT sequencing of genomes and transcripts can be acquired more efficiently (Chao et al. 2018). Transcriptome profiling has become an effective means for studying plant responses to stress, and it has been used widely to interpret how plants react to biotic and abiotic stress in various species (Deng et al. 2018; Ren et al. 2018; Zhu et al. 2018). In our early research we found that shoots of resistant plants contain much higher polyphenols and tannins, and much lesser insect numbers than susceptible plant shoots (Mei et al. 2018). In addition our feeding 
77 studies showed that Agrilus mali feeding preference on the leaves of susceptible plants compared

78 to resistant one (Fig S1). So we decided to analyze the specific mechanisms involved in the

79 process through full-length transcriptome technology. In this study

80 analyses of the transcriptome of shoot phloem from resistant and susceptible strains of $M$

81 sieversii were carried out using RNA-seq technologies aiming to identify

82 differential genes that may be involved in plant-insect interaction.

83 Materials and Methods

84 Plant materials

85 On June 25, 2017, 50 shoots from 10 selected trees (five resistant and five susceptible) were

86 collected from the wild $M$ sieversii population located in Gailiangchang $\left(\mathrm{N}^{\circ} 3^{\circ} 22, \mathrm{E} 83^{\circ} 34\right.$, the

87 altitude 1,280-1,450 m), Xinyuan County, Xinjiang Autonomous Region. Field experiments

88 was approved by the research council of the Institute of Horticultural Crops,

Xinjiang Academy of Agricultural Sciences (approval number: XAASHC2016.02.08). The selected trees (resistant and susceptible) have been proved to be stable in their insect-interaction traits for years. All the trees were aged 45-60 years old.

Infection treatment

The apple buprestid beetles usually parasitize the phloem of branches. According to their life cycle, adults lay eggs in the phloem underneath the bark. The larvae begin feeding the follow spring. The damage caused by larval feeding peaks in July and lasts until September. Ten mature larvae (one larvae for per shoot) were inserted into each tree through a simulated pore canal that created by $\mathrm{T}$ shape cutting of phloem. After a period of ten days, phloem were sampled from the infested and uninfested parts of the resistant and susceptible trees after the areas from the parts $>3 \mathrm{~cm}$ away from the original mechanical damage had healed. (The sampled spots with obvious infection were chosen). Samples were frozen immediately in liquid nitrogen and stored at $-80^{\circ} \mathrm{C}$ before RNA extraction. Samples were named as follows: Insect-feeding resistant plant (IFR): Insect-feeding susceptible plants (IFS); Nonefeeding resistant plants (NFR); None-feeding susceptible plants (NFS).

\section{RNA extraction, cDNA library construction, and Illumina sequencing}

Total RNA was extracted from each sample using a RNA extraction kit (TissueLyzer; Qiagen, Valencia, CA, USA), according to manufacturer's instructions. RNA purity was checked using a Nano Photometer spectrophotometer (IMPLEN, CA, USA), degradation and contamination were monitored on a 1\% agarosegel, and integrity was assessed by an Agilent Bioanalyzer Nano 6000. Three $\mu \mathrm{g}$ of RNA was taken from each qualified sample and mixed. mRNA was purified from total RNA using poly-T oligo-attached magnetic beads. The sequencing library was prepared

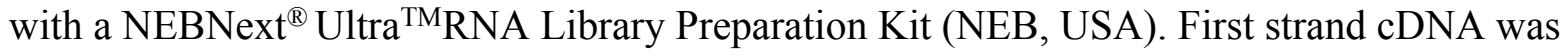
synthesized using a random hexamer primer and M-MuLV Reverse Transcriptase (RNase H-). 
113 DNA polymerase I and RNase $\mathrm{H}$ were added sequentially into the obtained first strand cDNA to

114 produce the second cDNA strand. The fragments in the library were purified using an

115 AMPureXP system (Beckman Coulter, Beverly, USA). The obtained double-stranded cDNA was

116 subjected to end repair, addition of an A-tail, and adaptor ligation, then AMPure XP beads were

117 used for size selection and, finally, PCR was adopted for enrichment to construct a cDNA library.

118 Library quality was assessed with an Agilent Bioanalyzer 2100 system. After qualification and

119 quantification of the library, short-read sequencing of each treatment $x$ phenotype combination

120 was performed using the Illumina HiSeqX-ten platform for RNA-Seq analysis.

121 Construction of single molecule real-time library and sequencing

122 PacBio RS II and Illumina sequencing were done on the same sample from the same site. We 123 mixed equal amounts of RNA and sequenced them. A SMARTer ${ }^{\text {TM }}$ PCR cDNA Synthesis Kit 124 was used to get full-length cDNA of mRNA, which was then screened with BluePippin ${ }^{\text {TM }}$ Size.

125 Three cDNA libraries (1-2k, 2-3k, 3-6k) were constructed. PCR was used again to amplify the

126 selected full-length cDNA. Then, they were repaired at the end, ligated with the SMRT

127 dumbbell-type adapters, and cleaved by exonuclease. A secondary screening was performed

128 using Blue Pippin to obtain the sequencing libraries. After the libraries were constructed, they

129 would quantify accurately using Qubit2.0, and the fragment size of libraries was detected using

130 Agilent 2100. Only those complying with a standard could be sequenced. Long-read sequencing

131 was performed using PacBio RS II platform.

132 Preprocessing of SMRT reads

133 Reads of Insert (ROI) sequences were extracted using the following criteria: $>=0$ full-pass 134 reading of the polymerase and sequence accuracy $>=75$. Readings below 50 bp in length were 135 discarded. ROI sequences were divided into two categories: full length sequences (that included 136 a $5^{\prime}$ primer, a $3^{\prime}$ primer, and a polyA tail) and non-full-length sequences. We used the ICE 137 algorithm of SMRT Analysis (v2.3.0) for iterative clustering, which collected similar sequences 138 (i.e., multiple copies of the same transcript) into the same cluster. The parameters used are the 139 default parameters. We used quiver to correct consensus sequences of each cluster, which 140 yielded high quality transcripts (HQ) with an accuracy $>99 \%$ (Korlach et al. 2010). In this 141 project, the low-quality consensus sequences obtained from each sample were corrected using 142 the corresponding Illumina RNA seq data through proof read to improve sequence accuracy 143 (Hackl et al. 2014). PacBio RS II and Illumina sequencing data are deposited at the National 144 Center for Biotechnology Information (NCBI) database (NCBI Sequence Read Archive 145 SRP199016: https://www.ncbi.nlm.nih.gov/sra/?term=SRP199016).

146 Function annotation and classification

147 The annotation of transcriptome sequences were performed using the following seven databases:

148 Nr (NCBI non-

Peer] reviewing PDF | (2019:09:41736:4:0:NEW 19 Mar 2020) 
149 redundant protein sequences), Pfam (Protein family), KOG/COG (eukaryotic Ortholog Groups a 150 nd Clusters of Orthologous Groups of proteins), Swiss-

151 Prot (A manually annotated and reviewed protein sequence database), KEGG (Kyoto Encyclope 152 dia of Genes and Genomes) and GO (Gene Ontology). The read numbers were transformed to FP

$153 \mathrm{KM}$ (fragments per kilobase of transcript sequence per millions base pairs sequenced) value for $\mathrm{g}$ 154 ene expression quantification.

155

156

157

158

159

160

161

162

163

164

165

166

167

168

169

170

171

172

173

174

175

176

177

178

179

180

181

182

183

184

185

\section{Real-time quantitative PCR verification}

We applied qRT-PCR technology to validate the results of the differential expression analysis. Insect-resistant related genes with stable and drastic expression differences were selected for expression verification. Total RNA from each sample was reverse-transcribed using a 5×All-In-One MasterMix (Cat\# G492, Abm, Canada) kit to synthesize the first strand of cDNA. The expression data in the Illumina HiSeq sequencing results were extracted for comparative analysis. Specific primers were designed using Primer-BLAST online software, and the melting curve was drawn to confirm PCR specificity. The quantitative PCR reaction conditions were as follows: $94{ }^{\circ} \mathrm{C}$ for $60 \mathrm{~s}$, followed by 45 cycles of $94{ }^{\circ} \mathrm{C}$ for $5 \mathrm{~s}, 60^{\circ} \mathrm{C}$ for $15 \mathrm{~s}$, and $72{ }^{\circ} \mathrm{C}$ for 10 s. SYBR was adapted to premix EvaGreen Express $2 \times$ qPCR MasterMix (Cat\# MasterMix-ES, Abm, Canada). The test was performed with apple MdTUB1 as the reference gene using LightCycler 96 (Roche, USA). Three biological replicates were set for each group, and we used three technical replicates for each biological replicate. Please see Table S1 for the sequence of qRT-PCR primers.

\section{Detection of secondary metabolites}

The plant material was stored at $-80^{\circ} \mathrm{C}$ and ground with a grinder(MM 400, Retsch) at $30 \mathrm{~Hz}$ for 1 minute. $100 \mathrm{mg}$ of the powder was extracted in $1.0 \mathrm{~mL}$ of methanol, during which the sample was rotated three times to mix the solvent. After the extraction, 12,000g was centrifuged for 15 minutes to obtain a supernatant. The obtained supernatant was blown dry with nitrogen gas in a $35^{\circ} \mathrm{C}$ heating mode, then, resuspended in a mixture of $30 \%$ methanol with vortexing, centrifuged at 12,000 $\mathrm{g}$ for 15 minutes, and the supernatant was collected and stored for analysis on an LC/MS. The content of secondary metabolites was detected by liquid chromatographymass spectrometry with reference to the method of Francescato, Pan and Wojakowska (Francescato et al. 2013; Pan et al. 2010; Wojakowska et al. 2013). In this experiment, all chemical reagents were of analytically pure or chromatographically pure preparations. Chemical standards are from BioBioPha (http://www.biobiopha.com/) and Sigma Aldrich (http://www.sigmaaldrich.com/united-states.html).

The analysis conditions were as follows: The sample extracts were analyzed using an

LC-ESI-MS/MS system (HPLC, Shim-pack UFLC Shimadzu CBM30A, www.shimadzu. com.cn/; MS, Applied Biosystems 4500 Q TRAP, www.appliedbiosystems.com.cn/). The analyti cal conditions were as follows: HPLC: column, Waters ACQUITY UPLC HSS T3 C18 (1.8 $\mu$ m, 
$2.1 \mathrm{~mm} * 100 \mathrm{~mm})$; solvent system, water $(0.04 \%$ acetic acid): acetonitrile $(0.04 \%$ acetic acid $)$; gr adient program, 95:5 V/V at $0 \mathrm{~min}, 5: 95 \mathrm{~V} / \mathrm{V}$ at $11.0 \mathrm{~min}, 5: 95 \mathrm{~V} / \mathrm{V}$ at $12.0 \mathrm{~min}, 95: 5 \mathrm{~V} / \mathrm{V}$ at 12. $1 \mathrm{~min}, 95: 5 \mathrm{~V} / \mathrm{V}$ at $15.0 \mathrm{~min}$; flow rate, $0.40 \mathrm{ml} / \mathrm{min}$; temperature, $40^{\circ} \mathrm{C}$; injection volume: $5 \mu$ l. The effluent was alternatively connected to an ESI-triple quadrupole-linear ion trap (Q TRAP)-MS.

For quantification of metabolites, standard solutions of different concentrations were prepared. Then mass spectrometry was used to analyze $0.1 \mathrm{ug} \cdot \mathrm{mL}^{-1}, 0.2 \mathrm{ug} \cdot \mathrm{mL}^{-1}, 0.5 \mathrm{ug} \cdot \mathrm{mL}^{-1}, 1.0 \mathrm{ug} \cdot \mathrm{mL}^{-1}$, $2.0 \mathrm{ug} \cdot \mathrm{mL}^{-1}, 5.0 \mathrm{ug} \cdot \mathrm{mL}^{-1}, 10.0 \mathrm{ug} \cdot \mathrm{mL}^{-1}$ standard solutions and samples.

\section{Correlation analysis of transcriptome and metabolome data}

By combining the metabolomics and transcriptomics data through the KEGG metabolic pathway, differentially expressed / accumulated genes and metabolites involved in the same biological process (KEGG Pathway) can be identified quickly.

\section{Statistical analysis}

All the experimental data obtained were analyzed using SPSS 17 software (SPSS, Inc. Chicago, $186 \mathrm{IL}, \mathrm{USA})$ and indicated by means $\pm(\mathrm{n}=3)$ standard deviation (SD). Data were analyzed usin g One-way ANOVA and Duncan test at a significance level of $\mathrm{p}<0.05$.

\section{Results}

\section{Morphological and physiological differences between resistant and susceptible plants} Susceptibility to the Agrilus mali invasion, soluble sugar, polyphenol as well as tannin contents of $M$ sieversii is shown (Fig. 1) to illustrate the difference between resistant and susceptible plants. Greater contents for polyphenol and tannin were observed in resistant plant shoots compared to susceptible plants (Fig.1A-B). However infection rate (number of insects) and soluble sugar contents in the shoots of susceptible plants were significantly higher than resistant plants (Fig.1D). As the important secondary metabolites in plants, polyphenols and tannin are closely related to the disease resistance of plants, also play an important role in plant-insect interaction. Therefore higher polyphenol and tannin contents in resistant plant shoots may be related to resistance of these plants to Agrilus mali invasion. In the later section, we will focus on pathways involved biosynthesis of secondary metabolites such phenolic acids and plant-pathogen interaction.

\section{PacBio RS II and Illumina HiSeq sequencing}

Transcriptome sequencing was performed using Illumina HiSeq X ten and PacBioRS II. First, all RNA samples were tested with an Illumina HiSeq X ten, which resulted in a total of $130.06 \mathrm{~Gb}$ Clean Data and Q30 up to 93.38\% (Table S2). Then, an equal amount of the 12 RNA samples were mixed together for PacBio library preparation and sequencing. The full-length cDNA of the poly(A) + RNA sample was standardized and SMRT-sequenced using the PacBio RS II platform. The number of sequencing cells was two for a $1-2 \mathrm{~K}$ library, and one each for the $2-3 \mathrm{~K}$ and $3-6 \mathrm{~K}$ 
222 libraries. A total of 122,103 full-length non-chimeric (FLNC) reads (Table 1) and 275, 586 ROI

223 (reads of insert) (Table 2) were obtained. SMRT Analysis software was employed to cluster the 224 full-length sequences, and a total of 64,692 consensus transcript sequences were generated. Non-

225 full-length sequence correction produced 47,181 high-quality, full-length transcript sequences 226 and 17,511 low-quality sequences, which were corrected using Illumina Hiseq data (Table S3).

227 The sequences were clustered iteratively using SMRT Analysis (v2.3.0) software and the ICE 228 (Iterative Clustering for Error Correction) algorithm. In this experiment, we received a total of 229 64,692 F01 consensus sequences with HQ (High-quality) and LQ (low-quality) transcripts that 230 were obtained from different libraries (Table S3). At least 27,230 HQ transcripts were obtained 231 from the 1-2 kb library, and 9,895 HQ were obtained from the 2-3Kb library. In addition, the LQ 232 consensus sequences were corrected by Illumina RNA seq platform using proof reads software to 233 improve their accuracy (Hackl et al. 2014).

\section{Verification of RNA-Seq gene expression}

To confirm the reliability of the RNA-Seq data, synthetic pathways, transcription factors, and important genes related to plant defense were screened from the annotation library of RNA-Seq DEGs data, and 18 of them were selected for real-time quantitative PCR (qRT-PCR). By doing so, effects of infection on expression of defense-related genes, and the accuracy of RNA-Seq data were verified (Fig 2). Quantitative results showed that all 18 genes responded to infection intensively, which included 13 up-regulated and five down-regulated genes. Most changes in gene expression were consistent with the expression patterns in RNA-Seq results, which confirmed the reliability of Illumina HiSeq sequencing in this experiment. Furthermore, expression level of a gene annotated as protein TIFY9 increased significantly after insect induction, which exhibited more than a 30-fold up-regulation in resistant strains and a more than 10-fold up-regulation in the control. This indicated the active participation of TIFY genes in the regulation of an insect-resistant response.

Functional annotation and selection of candidate DEGs related to plant pest resistance In order to identify genes respond to Agrilus mali invasion in resistant plants, firstly we select common DEGs in NFS vs NFR (A), IFS vs IFR (B), NFR vs IFR(C) and NFS vs IFS (D) comparison groups, respectively. Then we used these common DEGs to build the Venn-diagram (Fig S2). It is evident from the Venn graph that 266 DEGs respond in resistant genotype compared to the susceptible one and the control (non-feeding) samples(Fig. 3A). These DEGs mainly enriched in the pathways of phenylalanine metabolism, degradation of ammonic acids, Flavonoid biosynthesis, phenylpropanoid biosynthesis, terpenoid backbone biosynthesis, degradation of aromatic compounds, fatty acid metabolism, phosphatidylinositol signaling, plant hormone signal transduction pathway, plant-pathogen interaction etc. (Fig. 3B). Among these DEGs 240 of 266 were up-regulated and 26 DEGs were down-regulated in resistant plants after 
258

259

260

261

262

263

264

265

266

267

268

269

270

271

272

273

274

275

276

277

278

279

280

281

282

283

284

285

286

287

288

289

290

291

292

293

infection by Agrilus mali indicating that these genes may participated in plant-insect interaction (Fig. S3). In addition majority of these genes enrichen in secondary metabolic pathways verifying the important role of secondary metabolites in plant defense system. Among these 266 DEGs, we mined 19 DEGs that enriched in phenylalanine metabolic pathway, flavonoid biosynthetic pathway and plant-pathogen interaction. These selected 19 genes will be further analyzed in following section.

\section{Correlation analysis of transcriptome and metabolome}

Ten days after insect feeding, we analyzed a group of bioactive secondary metabolites such as phenolic acids, alkaloids, flavones, salicylic acid, chlorogenic acid and benzoic acid that involved in plant defense system (Figure S4). Among these metabolites, only 3 phenolic acids differentially accumulated in different samples (Figure.4A-C). Trans-cinnamic acid and caffeic acid content increased with 1.3-fold, 1.1-fold and 7.3-fold, 2.8-fold in susceptible and resistant plants, respectively. The fold changes of these two metabolites were much higher in resistant plant than in susceptible plant, indicating that these metabolites may play critical role in plantinsect interaction. Through correlation analysis, we found that genes encoding enzymes (PAL, $\mathrm{C} 4 \mathrm{H}$ ) responsible for biosynthesis of these four phenolic acids were differentially express in susceptible and resistant plants (Figure.4D). In accordance with metabolites content, expressions of correlated genes were much higher in resistant plants compared to susceptible plants.

\section{Discussion}

The plant defense response to insect damage involves relatively complex regulatory pathways. Under biotic stress, stress response of plants can be understood through transcriptome analysis and identification of relevant candidate genes (Hettenhausen et al. 2017; Zhuang et al. 2018). To obtain a higher-quality read length, we introduced real-time SMRT and corrected the results by combing Illumina HiSeq data to improve the accuracy of transcriptome analysis. Using four cells, $10.52 \mathrm{~Gb}$ clean data were obtained, which included 275,586 ROIs and 122,103 FLNC sequences (Table 1, 2). Illumina HiSeq sequencing data were used to correct 17,511 low-quality sequences (Table S3). Among the 9,598 DEGs, the genes (266) respond to Agrilus mali infection in resistant plants were mainly analyzed (Table S4). In present research we found that resistant plants contain significantly higher polyphenols and tannins than susceptible plants (Fig 1). In addition in our metabolomics we identify three phenolic acids (trans-cinamic acid, caffeic acid and ferulic acid) that differently accumulated in resistant and susceptible plants. All of these three metabolites were significantly increased in both two samples after Agrilus mali infection. Interestingly, fold changes of trans-cinamic acid and caffeic acid in resistant plants were much higher than in susceptible ones after Agrilus mali infection indicating their importance in plant-insect interaction (Fig 4). Among these 266 DEGs we mined 19 genes that involved in secondary metabolism. Further correlation analysis between DEGS and metabolites revealed that majority of these 19 genes encode enzymes 
294 responsible for synthesis of above mentioned phenolic acids verifying the importance of these 295 phenolic acids in plant defense system.

296 Phenylalanine lyase (PAL) catalize phenylalanine ammonia to form cinnamic acid. it is reported 297 that insect attack induce higher expression of $P A L$, accompanied by changing related hormone 298 levels and metabolites (Chaman et al. 2003; Han et al. 2009). In accordance with these findings, in

299

300

301

302

303

304 our experiment we found that $P A L$ was strongly induced by Agrilus mali infection and correspondingly trans-cinamic acid content were also increased exponentially. After entering the phenylpropane metabolic pathway, $P A L$ further mediates the synthesis of flavonoids, lignin, anthocyanins, plant antitoxins and plant hormones that regulate plant-insect interaction. Cinnamate 4-hydroxylase $(\mathrm{C} 4 \mathrm{H})$, belonging to CYP73 family of P450 super family, responsible for catalyzing trans-cinamic acid to form $p$-coumaric acid. Studies have shown that plant-mediated RNAi silencing of the $\mathrm{C} 4 \mathrm{H}$ monooxygenase gene significantly increased the tolerance of the larvae to gossyphenol, and the larvaes grew slowly when feeding on $\mathrm{C} 4 \mathrm{H}$ over expressed plant materials (Mao et al. 2007). Similarly we identified five $C 4 H$ genes that strongly induced in resistant plants compared to susceptible plants. $\mathrm{C} 4 \mathrm{H}$ activity requires molecular oxygen and NADPH supplied by the NADPH generation system to produce activity. Plant NADPH oxidase, also known as $R B O H s$, is responsible for the production of signal ROS in the plant defense response (Marino et al. 2012). In Arabidopsis over expression of $R B O H D$ and $R B O H F$ increased RBOH/NADPH oxidase dependent ROS production and signaling in Arabidopsis immunity (Torres et al. 2001). In our study we found that one transcripts of $R B O H F$ up-regulated. In our study, $R B O H F$ gene expression was up-regulated with 50-fold and 3.3-fold respectively in both resistant and susceptible plants after infection suggesting that $R B O H F$ might mediate ROS signaling in response to external stimulus and activate plant defense system.

Jasmonate is an important plant hormone, which is necessary for acclimation of plants to adversity condition. JAZs transcription factors, belongs to a larger family of plant specific TIFY proteins, respond to the jasmonoyl-L-isoleucine (JA-Ile), which each members have different in response patterns to injury induction (Chung et al. 2009; Thireault et al. 2015). In this experiment, we identified three transcripts of TIFY9 and all of these three genes were up-regulated after insect infection in both resistant and susceptible plants. TIFY family genes are important regulatory genes for JA accumulation, so it is speculated that TIFY proteins play an important role in plantinsect interaction. However functions of TIFY family members are still unclear and further study need to be done in the future works.

\section{Conclusions}

This study shows that the contents of polyphenols, tannins, and soluble sugars in $M$ sieversii resistant strains are significantly different from those in the susceptible strains. Furthermore, 266 DEGs associated with insect-resistance were screened from transcriptome (240 expressions were 
330 up-regulated and 26 expressions were down-regulated). There are 19 accumulated DEGs

331 associated with insect resistance in the phenylalanine metabolic pathway, flavonoids and

332 jasmonate biosynthetic pathway. Among them, C4H, RBOHF, PAL and other genes are involved

333 in the regulation of phenylpropane pathway. Targeted metabolomics results also confirm this.

334 After the pest feeding, the trends in the secondary metabolites such as trans-cinnamic acid,

335 caffeic acid and ferulic acid were consistent with the expression of the above genes. Therefore, it

336 is likely that genes such as $\mathrm{C} 4 \mathrm{H}, \mathrm{RBOHF}$, and PAL mediate phenylpropane signaling and induce

337 the synthesis of downstream secondary metabolites, resulting in the insect-resistant phenotype.

338 Data Availability

339 The following information was supplied regarding data availability:

340 The raw data for all the figures are available in a Supplemental File.

\section{Supplemental Information}

342 Supplementary Table 1: qRT-PCR primers used in this study.

343 Supplementary Table 2: Illumina HiSeq Sequencing Data for samples collected from healthy and 344 infested wild apple trees in China.

345 Supplementary Table 3: ICE clustering result for samples collected from wild apple trees in 346 China.

347 Supplementary Table4: Functional annotation and selection of candidate 266 DEGs related to 348 plant pest resistance.

349 Supplementary Figure 1: Phenotypic data of Malus sieversii after infection with Agrilus mali.

350 Supplementary Figure 2: Common DEGs in NFS vs NFR (A), IFS vs IFR (B), NFR vs IFR(C) 351 and NFS vs IFS (D) comparison groups, respectively.

352 Supplementary Figure 3: Up-regulating and down-regulating DEGs Venn diagram.

353 Supplementary Figure 4: Insect-resistant metabolites detected in targeted metabolome.

354 Acknowledgments

355 Collection of experimental materials was supported by Ili Forestry Research Institute. We would 356 like to thank Thomas A. Gavin, Professor Emeritus, Cornell University, for help with editing this 357 paper. 


\section{References}

Barah P, and Bones AM. 2015. Multidimensional approaches for studying plant defence against insects: from ecology to omics and synthetic biology. Journal of Experimental Botany 66:479-493. doi:10.1093/jxb/eru489

Chaman ME, Copaja SV, and Argando VH. 2003. Relationships between Salicylic Acid Content, Phenylalanine Ammonia-lyase (PAL) Activity, and Resistance of Barley to Aphid Infestation. Journal of Agricultural and Food Chemistry 51:2227-2231.

Chao Y, Yuan J, Li S, Jia S, Han L, and Xu L. 2018. Analysis of transcripts and splice isoforms in red clover (Trifolium pratense L.) by single-molecule long-read sequencing. BMC Plant Biology 18:300. doi:10.1186/s12870-018-1534-8

Chung HS, and Howe GA. 2009. A critical role for the TIFY motif in repression of jasmonate signaling by a stabilized splice variant of the JASMONATE ZIM-domain protein JAZ10 in Arabidopsis. The Plant cell 21:131-145. doi:10.1105/tpc.108.064097

Deng Y, Zheng H, Yan Z, Liao D, Li C, Zhou J, and Liao H. 2018. Full-Length Transcriptome Survey and Expression Analysis of Cassia obtusifolia to Discover Putative Genes Related to Aurantio-Obtusin Biosynthesis, Seed Formation and Development, and Stress Response. International Journal of Molecular Sciences 19:2476. doi:10.3390/ijms19092476

Duan N, Bai Y, Sun H, Wang N, Ma Y, Li M, Wang X, Jiao C, Legall N, Mao L, Wan S, Wang K, He T, Feng S, Zhang Z, Mao Z, Shen X, Chen X, Jiang Y, Wu S, Yin C, Ge S, Yang L, Jiang S, Xu H, Liu J, Wang D, Qu C, Wang Y, Zuo W, Xiang L, Liu C, Zhang D, Gao Y, Xu Y, Xu K, Chao T, Fazio G, Shu H, Zhong GY, Cheng L, Fei Z, and Chen X. 2017. Genome re-sequencing reveals the history of apple and supports a two-stage model for fruit enlargement. Nature Communications 8:249. doi:10.1038/s41467-017-00336-7

Ebel C, BenFeki A, Hanin M, Solano R, and Chini A. 2018. Characterization of wheat (Triticum aestivum) TIFY family and role of Triticum Durum TdTIFY11a in salt stress tolerance. PLoS One 13:e0200566. doi:10.1371/journal.pone.0200566

Forsline PL, Aldwinckle HS, Dickson EE, Luby JJ, and Hokanson SC. 2010. Collection, Maintenance, Characterization, and Utilization of Wild Apples of Central Asia. Horticultural Reviews 29:1-61.

Francescato LN, Debenedetti SL, Schwanz TG, Bassani VL, and Henriques AT. 2013. Identification of phenolic compounds in Equisetum giganteum by LC-ESI-MS/MS and a new approach to total flavonoid quantification. Talanta 105:192-203. doi:10.1016/j.talanta.2012.11.072

Hackl T, Hedrich R, Schultz J, and Forster F. 2014. proovread: large-scale high-accuracy PacBio correction through iterative short read consensus. Bioinformatics 30:3004-3011. doi:10.1093/bioinformatics/btu392

Han Y, Wang Y, Bi J-L, Yang X-Q, Huang Y, Zhao X, Hu Y, and Cai Q-N. 2009. Constitutive and Induced Activities of Defense-Related Enzymes in Aphid-Resistant and Aphid-Susceptible Cultivars of Wheat. Journal of Chemical Ecology 35:176-182.

Hettenhausen C, Li J, Zhuang H, Sun H, Xu Y, Qi J, Zhang J, Lei Y, Qin Y, Sun G, Wang L, Baldwin IT, and Wu J. 2017. Stem parasitic plant Cuscuta australis (dodder) transfers herbivory-induced signals among plants. Proceedings of the National Academy of Sciences of the United States of America 114:E6703-E6709. doi:10.1073/pnas.1704536114

Howe GA, and Jander G. 2008. Plant immunity to insect herbivores. Annual Review of Plant Biology 59:41-66. 
doi:10.1146/annurev.arplant.59.032607.092825

Korlach J, Bjornson KP, Chaudhuri BP, Cicero RL, Flusberg BA, Gray JJ, Holden D, Saxena R, Wegener J, and Turner SW. 2010. Chapter 20 - Real-Time DNA Sequencing from Single Polymerase Molecules. Methods in Enzymology 323:431-455.

Liu Q, Wang X, Tzin V, Romeis J, Peng Y, and Li Y. 2016. Combined transcriptome and metabolome analyses to understand the dynamic responses of rice plants to attack by the rice stem borer Chilo suppressalis (Lepidoptera: Crambidae). BMC Plant Biology 16:259. doi:10.1186/s12870-016-0946-6

Ma X, Cai Z, Liu W, Ge S, and Tang L. 2017. Identification, genealogical structure and population genetics of Salleles in Malus sieversii, the wild ancestor of domesticated apple. Heredity 119:185-196. doi:10.1038/hdy.2017.28

Mao Y-B, Cai W-J, Wang J-W, Hong G-J, Tao X-Y, Wang L-J, Huang Y-P, and Chen X-Y. 2007. Silencing a cotton bollworm P450 monooxygenase gene by plant-mediated RNAi impairs larval tolerance of gossypol. Nature Biotechnology 25:1307-1313. doi:10.1038/nbt1352

Marino D, Dunand C, Puppo A, and Pauly N. 2012. A burst of plant NADPH oxidases. Trends in Plant Science 17:9-15. doi:10.1016/j.tplants.2011.10.001

Mei C, Yan P, Ai S, Ma K, Han L, Xu Z, Zhong H, Diao Y, and Wang J. 2016. The Relationship between Bark Thickness and Branch Roughnesson Agrilusmali Damage in Xinjiang Wild Apple. Journal of Agricultural Science and Technology 18:24-30 (in Chinese with English abstract).

Mei C, Yan P, Maimaiti A, Han L, Zhang Y, Ma K, and Wang J. 2018. Links Between Nutrients, Secondary Metabolites of Xinjiang Wild Apple and Agrilus mali Mats Resistance. Journal of Agricultural Science and Technology 20:26 - 33 (in Chinese with English abstract).

Pan X, Ruth W, and Wang X. 2010. Quantitative analysis of major plant hormones in crude plant extracts by highperformance liquid chromatography-mass spectrometry. Nature Protocols 5:986-992.

Ren P, Meng Y, Li B, Ma X, Si E, Lai Y, Wang J, Yao L, Yang K, Shang X, and Wang H. 2018. Molecular Mechanisms of Acclimatization to Phosphorus Starvation and Recovery Underlying Full-Length Transcriptome Profiling in Barley (Hordeum vulgare L.). Frontiers in Plant Science 9:500. doi:10.3389/fpls.2018.00500

Thireault C, Shyu C, Yoshida Y, St Aubin B, Campos ML, and Howe GA. 2015. Repression of jasmonate signaling by a non-TIFY JAZ protein in Arabidopsis. The Plant journal 82:669-679. doi:10.1111/tpj.12841

Torres MA, Dangl JL, and Jones JDG. 2001. Arabidopsis gp91phox homologues AtrbohD and AtrbohF are required for accumulation of reactive oxygen intermediates in the plant defense response. Proceedings of the National Academy of Sciences 99:517-522. doi:10.1073/pnas.012452499

War AR, Paulraj MG, Ahmad T, Buhroo AA, Hussain B, Ignacimuthu S, and Sharma HC. 2012. Mechanisms of plant defense against insect herbivores. Plant Signal Behav 7:1306-1320. doi:10.4161/psb.21663

Wojakowska A, Piasecka A, García-López PM, Zamora-Natera F, and Stobiecki M. 2013. Structural analysis and profiling of phenolic secondary metabolites of Mexican lupine species using LC-MS techniques. Phytochemistry 92:71-86.

Xia W, Yu H, Cao P, Luo J, and Wang N. 2017. Identification of TIFY Family Genes and Analysis of Their Expression Profiles in Response to Phytohormone Treatments and Melampsora larici-populina Infection in Poplar. Frontiers in Plant Science 8:493. doi:10.3389/fpls.2017.00493

Xie Y, Chen P, Yan Y, Bao C, Li X, Wang L, Shen X, Li H, Liu X, Niu C, Zhu C, Fang N, Shao Y, Zhao T, Yu 
J, Zhu J, Xu L, van Nocker S, Ma F, and Guan Q. 2018. An atypical R2R3 MYB transcription factor increases cold hardiness by CBF-dependent and CBF-independent pathways in apple. The New Phytologist 218:201-218. doi:10.1111/nph.14952

Yi Z, Liu D, Cui X, and Shang Z. 2016. Morphology and Ultrastructure of Antennal Sensilla in Male and Female Agrilus mali (Coleoptera: Buprestidae). Journal of Insect Science 16:86. doi:10.1093/jisesa/iew073

Zhu C, Li X, and Zheng J. 2018. Transcriptome profiling using Illumina- and SMRT-based RNA-seq of hot pepper for in-depth understanding of genes involved in CMV infection. Gene 666:123-133. doi:10.1016/j.gene.2018.05.004

Zhuang H, J L, J S, C H, MC S, G S, C Z, J L, D S, and J W. 2018. Aphid (Myzus persicae) feeding on the parasitic plant dodder (Cuscuta australis) activates defense responses in both the parasite and soybean host. New Phytologist 218:1586. 


\section{Figure 1}

Differences in biochemical and infection rates between resistance and susceptible strains

A-C: Spectrophotometric detection of the contents of soluble sugars, tannins, and polyphenols in the phloem of branches. D: Number of bugs parasitic on resistance and susceptible strains. In this experiment, 10 branches with a length of $50 \mathrm{~cm}$ in each strain, then peel off the phloem and count the number of bugs under the bark.

A

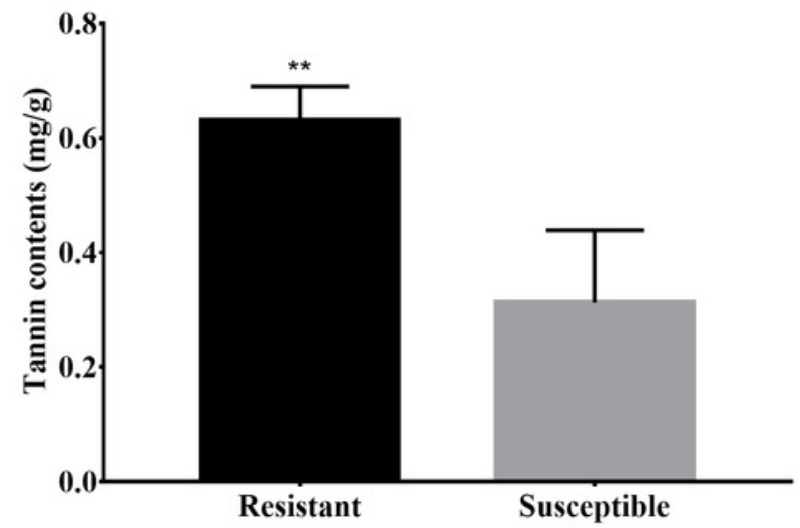

C

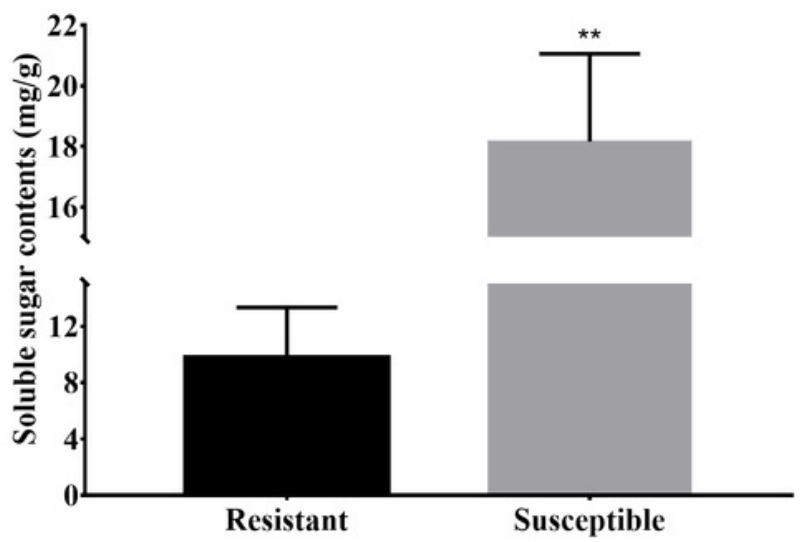

B

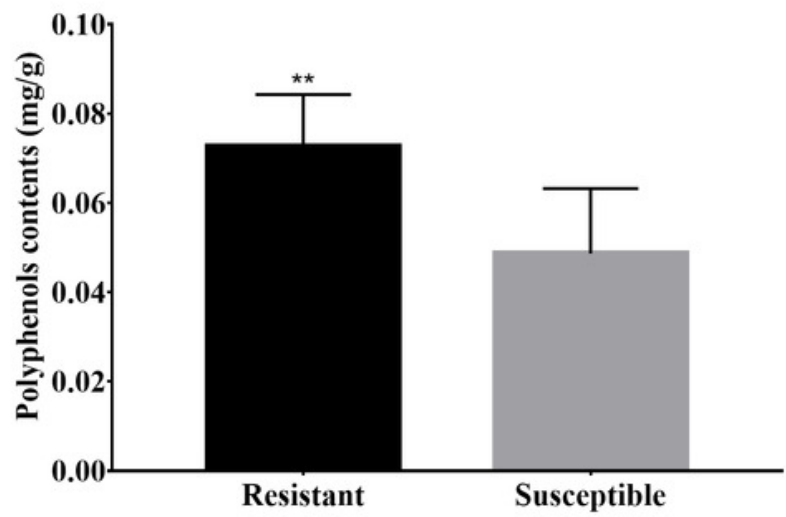

D

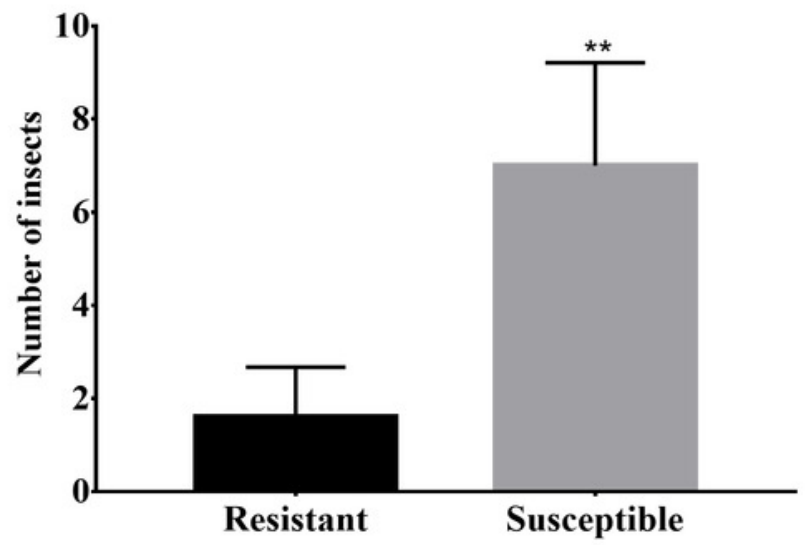


Figure 2

qRT-PCR used to verify expression of biotic stress-related genes in Xinjiang Wild Apple

A: qRT-PCR data. B: FPKM values obtained from the RNA-Seq data. Heat maps were produced using standardized figures that were transformed to a value between 0.0 and 1.0 by Min-Max normalization method.

A

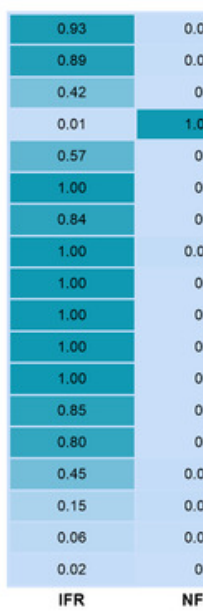

qPCR

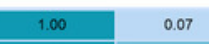

\begin{tabular}{|c|c|}
\hline 1.00 & 0.07 \\
\hline 1.00 & 0.49 \\
\hline 1.00 & 0 \\
\hline 0.11 & 0.36 \\
\hline 1.00 & 0.26 \\
\hline
\end{tabular}

\begin{tabular}{l|l|l|l|l}
\hline 1.00 & 0.26 \\
\hline 0.89 & 0.01
\end{tabular}

1.00

0.26

0.59

0.22

0.38

0.17

\begin{tabular}{l}
0.17 \\
1.00 \\
\hline 1.00
\end{tabular}

1.00

0.55

0.55

0.56

0.21

IFS NFS

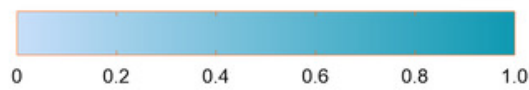

B

Protein TIFY 9

Trans-cinnamate 4-monooxygenase

Branched-chain-amino-acid aminotransferase 7

Ethylene-responsive transcription factor ERF073

Salicylic acid-binding protein 2

Ethylene-responsive transcription factor ABR1

G-type lectin S-receptor-like serine/threonine-protein kinase LECRK3

L-type lectin-domain containing receptor kinase 4.1

Cationic peroxidase 1

U-box domain-containing protein 21

ATP-dependent 6-phosphofructokinase 3

Indole-3-acetic acid-amido synthetase $\mathrm{GH} 3.1$

L-type lectin-domain containing receptor kinase 7.1

Serine/threonine-protein kinase EDR1

Histone deacetylase HDT1

LRR receptor-like serine/threonine-protein kinase

Protein LOL1

Alpha-amylase 2

\begin{tabular}{|c|c|c|c|}
\hline 1.00 & 0.10 & 0 & 0 \\
\hline 1.00 & 0.05 & 0.24 & 0.16 \\
\hline 1.00 & 0.01 & 0 & 0 \\
\hline 1.00 & 0.12 & 0.19 & 0.08 \\
\hline 1.00 & 0.09 & 0.24 & 0.11 \\
\hline 1.00 & 0.02 & 0.21 & 0 \\
\hline 1.00 & 0.02 & 0.12 & 0.03 \\
\hline 1.00 & 0.09 & 0.19 & 0.03 \\
\hline 1.00 & 0.02 & 0.02 & 0.03 \\
\hline 1.00 & 0.02 & 0.06 & 0.01 \\
\hline 1.00 & 0.06 & 0.15 & 0.03 \\
\hline 1.00 & 0 & 0.03 & 0 \\
\hline 1.00 & 0.01 & 0.15 & 0 \\
\hline 1.00 & 0 & 0.03 & 0 \\
\hline 0.06 & 0.25 & 0.30 & 1.00 \\
\hline 0 & 0.01 & 0.09 & 1.00 \\
\hline 0.08 & 0.23 & 0.26 & 1.00 \\
\hline 0.05 & 0.16 & 0.23 & 1.00 \\
\hline IFR & NFR & IFS & NFS \\
\hline
\end{tabular}

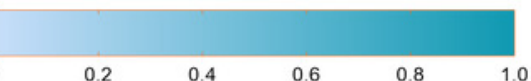




\section{Figure 3}

Screening and annotated functions of pest stress-responsive DEGs of Xinjiang Wild Apple

(A) NFS vs NFR normal refers to the comparison of healthy parts between the control and insect-resistant strains (blue). IFS vs IFR pet refers to the comparison of infected site between the control and insect-resistant strains (yellow). NFR vs IFR presents the comparison between healthy. parts and infected sites of insect-resistant strains (green). NFS vs IFS refers to the comparison between healthy parts and infected sites in the control (pink). (B) KEGG classification of the 266 differentially expressed Unigene. The ordinate is the name of the KEGG metabolic pathway, and the abscissa is the number of unigene annotated to the pathway.

A

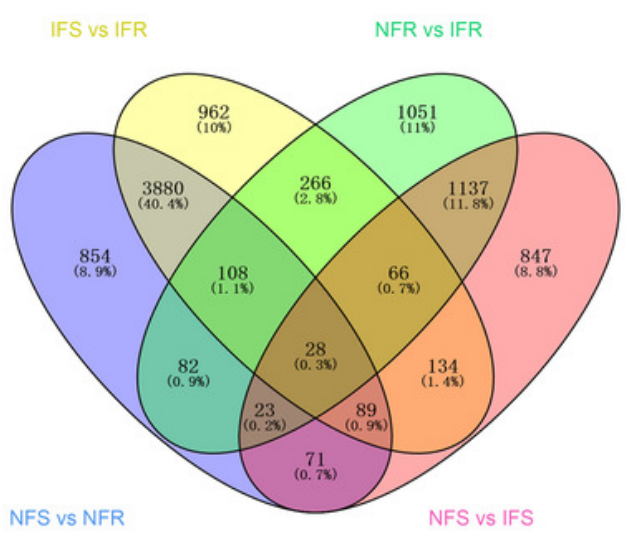

B

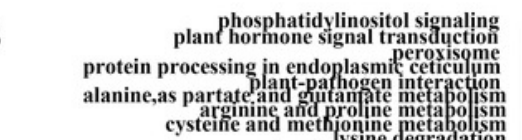

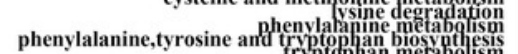

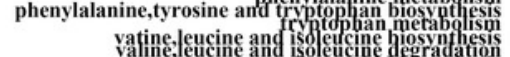

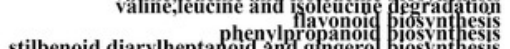

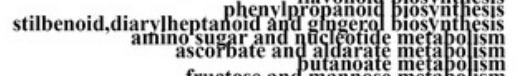

fructose and mannoase meetapo

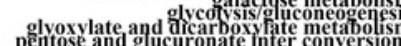

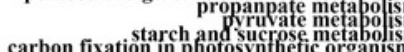

carbon fixation in starch and sucrose metabols $^{2}$

2-oxqcarboxylic acip merdbolis

degration of aroman metabolisp

biosynthesis of unsaturated fattt acid

tatty acid degradation
olycerolp matabolism

synthesis and deoration of biosynthesis ubiquinone and other terpenoig and coA biosynthes taurine and oputatime mecapo

sesquiter penoid and triterpenold piosynt jess

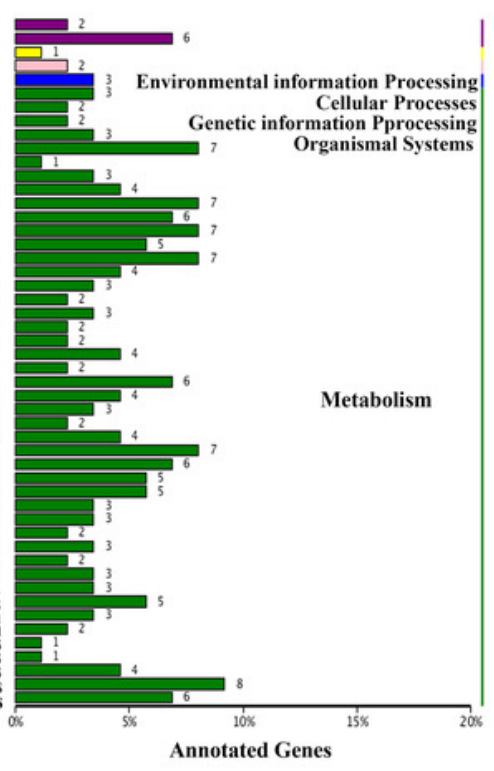




\section{Figure 4}

Corresponding anti-insect metabolic pathways and DEGs after Malus sieversii infection with Agrilus mali

A-C: Insect-resistant metabolites detected in targeted metabolome, such as trans- cinnamic acid, caffeic acid and ferulic acid. Non-Feeding represents uninfested samples, Feeding represents infested samples. D: Heat map of 19 insect resistance gene expression changes, and the 19 genes were selected from 266 DEGs. Heat maps were produced using standardized figures that were transformed to a value between 0.0 and1. 0 by Min-Max normalization method. 


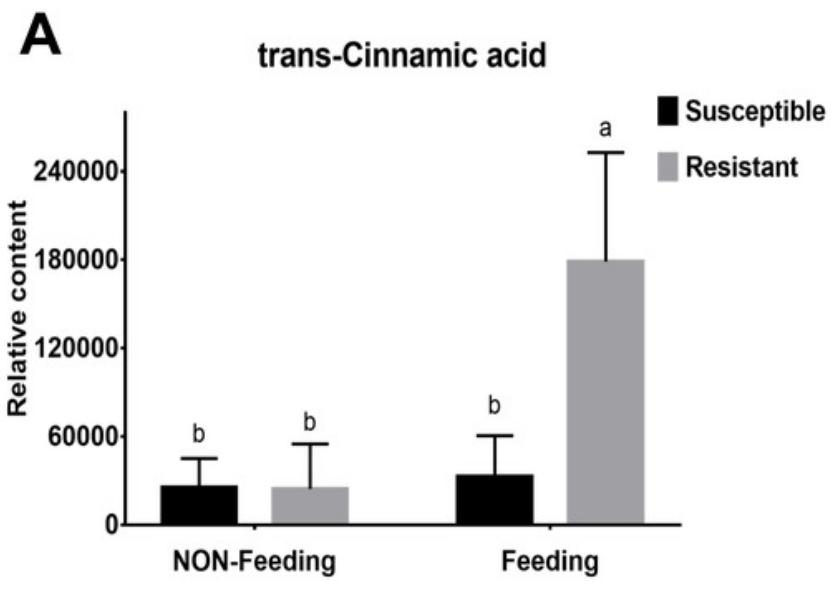

D
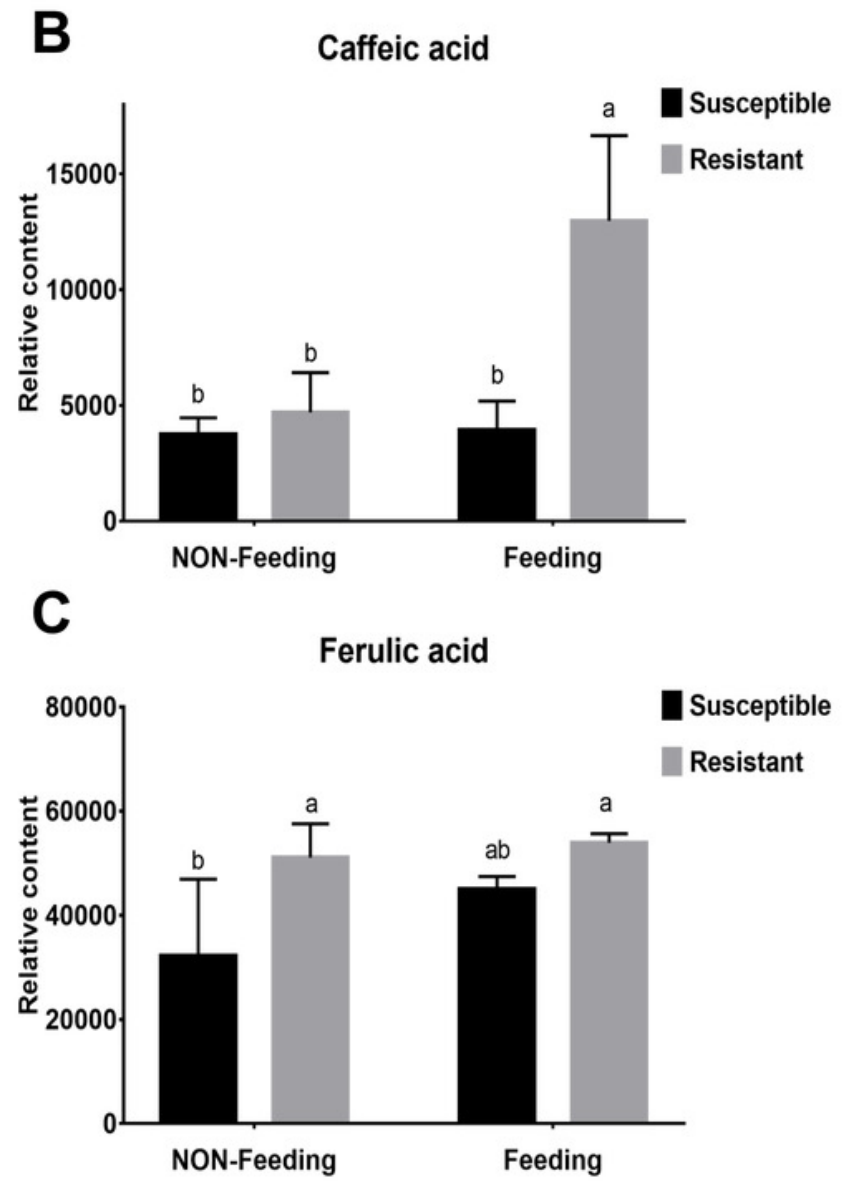

\begin{tabular}{|c|c|c|c|c|}
\hline 1.00 & 0.02 & 0.21 & 0.06 & Respiratory burst oxidase homolog protein $F$ \\
\hline 1.00 & 0.13 & 0.24 & 0.09 & Trens-innamate 4-monooxygenase \\
\hline 1.00 & 0.21 & 0.10 & 0.01 & WRKY transcipition factor WRKK24 \\
\hline 1.00 & 0 & 0.01 & 0 & Indole-3accetic acid-amido synthetase GH3.1 \\
\hline 1.00 & 0.05 & 0 & 0 & Phenylalanine ammonia-yyasel \\
\hline 1.00 & 0.02 & 0.02 & 0.03 & Cationic peroxidase 1 \\
\hline 0.06 & 0.65 & 0.24 & 1.00 & 3epif-dedexocathasterone 23-monooxygenase \\
\hline 1.00 & 0 & 0 & 0 & Heat shock cognate protein 80 \\
\hline 1.00 & 0.03 & 0.29 & 0.15 & Trens-innamate 4 -monooxygenase \\
\hline 1.00 & 0.09 & 0.16 & 0.08 & Protein TIFY 9 \\
\hline 1.00 & 0.02 & 0.02 & 0.05 & Auxin-responsive protein IAA11 \\
\hline 1.00 & 0.19 & 0.25 & 0.04 & Protein TFYY 9 \\
\hline 1.00 & 0 & 0.03 & 0 & Indole-3acetic acid-amido synthetase GH3.1 \\
\hline 1.00 & 0.04 & 0.23 & 0.17 & Trans-cinnamate 4-monooxygenase \\
\hline 1.00 & 0 & 0 & 0 & 4hydroxycoumanin synthase? \\
\hline 1.00 & 0.12 & 0.15 & 0.05 & Trans-cinnamate 4 monooxygenase \\
\hline 1.00 & 0.05 & 0.24 & 0.16 & Trans-cinnamate 4monooxygenase \\
\hline 1.00 & 0.05 & 0 & 0 & Chorismate Synthase, chloroplastic \\
\hline 1.00 & 0.10 & 0 & 0 & Protein TFYY \\
\hline IFR & NFR & $\mathbb{F S}$ & NFS & \\
\hline
\end{tabular}




\section{Table 1 (on next page)}

PacBio RS II sequencing data for samples collected from healthy and infested wild apple trees in China.

cDNA Size: the length of fragment in cDNA library; SMRT Cells $\square$ the amount of cells in all libraries; Polymerase Reads $\square$ the number of reads which were assembled by polymerase; FullLength Percentage (FL\%) : the percent of full-length reads in ROI. 


\begin{tabular}{ccccccc}
\hline $\begin{array}{c}\text { cDNA } \\
\text { size }\end{array}$ & $\begin{array}{c}\text { SMRT } \\
\text { Cells }\end{array}$ & $\begin{array}{c}\text { Polymerase } \\
\text { Reads }\end{array}$ & $\begin{array}{c}\text { Number of } \\
\text { full-length } \\
\text { reads }\end{array}$ & $\begin{array}{c}\text { Number of full- } \\
\text { length non- } \\
\text { chimeric reads }\end{array}$ & $\begin{array}{c}\text { Number of full- } \\
\text { length non- } \\
\text { chimeric reads }\end{array}$ & $\begin{array}{c}\text { Full-Length } \\
\text { Percentage } \\
\text { (FL\%) }\end{array}$ \\
\hline 1-2K & 2 & 300,584 & 72,228 & 71,914 & 71,914 & $48 \%$ \\
2-3K & 1 & 150,292 & 26,110 & 26,038 & 26,038 & $37 \%$ \\
$3-6 K$ & 1 & 150,292 & 24,165 & 24,151 & 24,151 & $45 \%$ \\
\hline
\end{tabular}

1

2

3

4

5

6

7

8

9

10

11

12

13

14

15

16

17

18

19

20

21

22

23

24

25

26

27

28

29 


\section{Table 2 (on next page)}

ROI sequences for samples collected from healthy and infested wild apple trees in China.

CDNA Size: the length of fragment in cDNA library, including primer, poly A and CDNA; Reads of Insert (ROI): the amount of ROI; Read Bases of Insert: the amount of bases in ROI; Mean Read Length of Insert: the quality average of ROI; Mean Number of Passes: the number of average sequencing times of ROI. 


\begin{tabular}{cccccc}
\hline $\begin{array}{c}\text { cDNA } \\
\text { size }\end{array}$ & $\begin{array}{c}\text { Reads of } \\
\text { Insert }\end{array}$ & $\begin{array}{c}\text { Read Bases } \\
\text { of Insert }\end{array}$ & $\begin{array}{c}\text { Mean Read } \\
\text { Length of Insert }\end{array}$ & $\begin{array}{c}\text { Mean Read } \\
\text { Quality of Insert }\end{array}$ & $\begin{array}{c}\text { Mean Number } \\
\text { of Passes }\end{array}$ \\
\hline 1-2K & 151,994 & $292,511,929$ & 1,924 & 0.93 & 13 \\
2-3K & 70,072 & $263,160,232$ & 3,755 & 0.9 & 7 \\
$3-6 \mathrm{~K}$ & 53,520 & $250,555,242$ & 4,681 & 0.88 & 5 \\
\hline
\end{tabular}

